\title{
$\operatorname{arCOS} D E S I G N$
}

\section{Apresentação Correspondências entre Design e Antropologia}

"O que pretendo dizer com correspondência [...] é o processo pelo qual seres e coisas literalmente co-respondem ou respondem entre si ao longo do tempo" (INGOLD, 2018, p.26, tradução nossa). Corresponder, na obra do antropólogo britânico Tim Ingold, é viver atencionalmente, e isso vem nos inspirando a construir modos de se fazer e pensar design por meio da antropologia. Os dois volumes deste dossiê apresentam uma consolidação de nossos esforços coletivos em sistematizar as correspondências entre o design e a antropologia. Nele, apresentamos algumas reflexões e referências que pontuam a aproximação de ambos os campos do saber, que vem sendo denominada de Design Anthropology (DA).

O percurso do campo de estudos em DA não é novo, e remonta às rupturas epistemológicas do design na década de 1970, quando, neste campo profissional, se ensaiava uma série de aproximações com a antropologia. Em um primeiro momento, através da ergonomia e dos estudos do antropólogo norte-americano Gordon W. Hewes, que, interessado pelos aspectos culturais e antropológicos das posições corporais, chamou a atenção dos designers para a necessária consideração da diversidade dos hábitos corporais no desenho de objetos dentro de uma civilização tecnológica. Essa aproximação não se restringiu, contudo, ao campo da ergonomia, espraiandose rapidamente para outras áreas do design. Indicava, sobretudo, um ponto de inflexão crucial para essa prática profissional, no sentido de um violento questionamento do papel social daqueles que tomam parte em projetos de desenvolvimento e produção de mercadorias industriais.

A emergência dessa revisão crítica da prática de projeto em design termina por levar os designers a uma aproximação com outros campos do saber, e, dentre eles, a antropologia, que parecia apresentar um outro modo 
de perceber e se aproximar da realidade social (CLARKE, 2011; WASSON, 2000). Nesse contexto, teve papel fundamental o livro "Design for a real world", publicado em 1971, pelo designer austríaco Victor Papanek, que percebia a antropologia como um antídoto à condição alienada à qual toda uma geração de designers desiludidos (CLARKE, 2011, p. 78) sentia estar aprisionada. Assim, as proposições de Papanek, no livro, explicitavam o que seria um dos grandes dilemas dos designers no século XX, a saber: sua inconsciência quanto às responsabilidades morais e sociais envolvidas em sua prática profissional. Em busca de "ecologias alternativas de design”, Papanek reuniu uma considerável bibliografia de referência em antropologia, fazendo também diversas incursões em campo, em meio a vários grupos sociais, dentre eles os esquimós, tema do artigo "Os melhores designers do mundo?", publicado no livro "Arquitectura e Design. Ecologia e Ética” (PAPANEK, 2002).

A partir dessa movimentação, a antropologia suscita cada vez mais interesse entre os designers, interesse que se desdobra em um conjunto diverso de articulações e intercâmbios entre as duas áreas, já repertoriado pelas antropólogas Christina Wasson no artigo "Ethnography in the field of design", publicado na revista "Human Organization" em 2000; por Alison J. Clarke na coletânea "Design Anthropology. Object Culture in 21st century", publicada em 2011; por Wendy Gunn e Jared Donovan, no livro "Design and Anthropology", de 2012; por Wendy Gunn, Ton Otto e Rachel Charlotte Smith, na publicação "Design Anthropology: theory and practice", de 2013; e por Rachel Charlotte Smith, Kasper Tang Vangkilde, Mette Gislev Kjaersgaard, Ton Otto, Joachim Halse e Thomas Binder, em "Design Anthropological Futures", de 2016; dentre outros.

Deve-se mencionar, também, a fundamental atuação da Research Network for Design Anthropology, que organizou uma série de seminários e conferências reunindo pesquisadores da área, sistematizando, assim, os temas e as abordagens desenvolvidos por acadêmicos em torno da aproximação entre design e antropologia. Essa rede é fruto de cooperações prévias entre diversas instituições de pesquisa em design na Dinamarca, a saber, o Codesign Research Centre, da Royal Danish Academy of Fine Arts, e o Participatory Innovation Research Centre, do Mads Clausen Institute for Product Innovation, University of Southern Denmark, que, por sua vez, foi parceiro da Danish Design School e de outras seis organizações no desenvolvimento da publicação "Rehearsing the future", de 2010, que apresenta os resultados de 
um projeto-piloto onde se buscava colocar em prática o que foi ali denominado de "Design Anthropological Innovation Model (DAIM)".

Uma importante discussão travada em meio a esse investimento de aproximação da antropologia em direção ao design se relaciona com as noções de prototipação e imaginação de futuros e com a referência às práticas de estúdios de projeto, temas desenvolvidos no livro "Designs for an anthropology of the contemporary", de Paul Rabinow e George Marcus (2008). Além dos autores já mencionados, destacam-se o antropólogo britânico Tim Ingold, que, em vários de seus trabalhos mais recentes (2000, 2007a, 2007b, 2011, 2013a, 2013b, 2015, 2016, 2018), discute as noções de desenho e projeto, dialogando com a educação, as artes, a arquitetura e o design, chegando a propor que se experimente praticar uma antropologia por meio do design (GATT, INGOLD, 2013); também deve-se notar a contribuição do francês Bruno Latour (2008), que, conclamando os designers a agirem com um pouco mais de cautela, formula a expressão "drawing things together". Ainda nesse trânsito de mão dupla, deve-se mencionar a já citada produção de diversos pesquisadores oriundos de vários países, organizada pela Research Network for Design Anthropology, rede de pesquisa articulada pelo antropólogo dinamarquês Joachim Halse, com financiamento do governo da Dinamarca.

Nosso objetivo, aqui, não é dar conta de todas as nuances de DA, mesmo porque são múltiplas as suas abordagens e as possibilidades de reflexão e ação, ainda em constituição; mas é o intuito apresentar pontos de partidas que compartilhamos entre nossas propostas de ação em dois núcleos de pesquisas instalados em duas universidades públicas brasileiras - o LADA, Laboratório de Design e Antropologia, da Escola Superior de Desenho Industrial, Universidade do Estado do Rio de Janeiro; e o NIDA, Núcleo de pesquisas em Inovação, Design e Antropologia, da Universidade Federal do Maranhão.

Como um importante resultado dessas trocas e trânsitos interdisciplinares e interinstitucionais, esta primeira parte do dossiê apresenta cinco artigos que constituem a produção recente de integrantes do LADA e do NIDA até o presente momento. O artigo Design Anthropology para muitos mundos possíveis, de autoria de Bibiana Serpa e Mariana Costard, ambas da ESDI/UERJ, abrem a edição, trazendo resultados de suas pesquisas de campo, visando propor uma visão ontológica do design, a partir do sul global, em uma perspectiva que critica as formas hegemônicas de se fazer e pensar design, criando novos e múltiplos caminhos possíveis, por meio do DA. 
O segundo artigo é de autoria de Maria Cristina Ibarra, da UFPE, intitulado Design como esperança no engajamento com um grupo de moradores de um bairro do Rio de Janeiro, no qual narra sua experiência como designer em um processo colaborativo mobilizado por um recém-formado coletivo para se pensar e enfrentar a violência no bairro de Santa Teresa, no Rio de Janeiro. Aborda o livro The method of Hope (2004), do antropólogo Hirokazu Miyazaki, e com ele dialoga sobre uma nova modalidade de engajamento etnográfico que aborda a esperança como método e não apenas como um objeto de análise. Esta discussão é fruto de sua tese realizada no PPDESDI.

No terceiro texto, Raiama Portela e Raquel Noronha trazem reflexões sobre os limites e alcances do uso de ferramentas em processos de DA, com o artigo Olhar, tocar e trocar: ferramenta em correspondência. As autoras, do PPGDg-UFMA, problematizam a ideia do protótipo em correspondência, pensando as relações entre seres e o ambiente vivido, a partir das intersubjetividades. Narram a pesquisa de campo com artesãs da fibra de buriti em Santa Maria, Alcântara, Maranhão, e questionam a ideia de empoderamento feminino e também realizam uma autorreflexão sobre seu próprio fazer enquanto designers e mediadoras de experimentos sociais.

O quarto artigo, Do uniforme ao desuniforme: Construção cartográfica de diferentes percepções a partir do vestuário estudantil, de autoria de Nayara Chaves Ferreira Perpétuo, Terezinha de Jesus Campos de Lima, Tiago Martins Azevedo e Jeane Sousa Santana, todos do IFMA, traz a discussão teórica do DA para a prática da construção de uma exposição colaborativa com o intuito de fortalecimento da memória institucional do antigo CEFET-MA e atual IFMA. Os fios da memória urdem a trama dos imaginários que, por meio dos uniformes, trazem os depoimentos de alunos do presente e do passado, e ajudam no processo de imaginação do futuro da instituição, com a construção de uma cartografia, que se constitui ao longo do período da exposição.

E finalizando esta edição, temos o texto Sobre amor, afeto e cidades, de autoria de Paula Oliveira Camargo, apresenta uma necessária e poética experiência sobre a escrita em DA, trazendo o olhar e as vivências cotidianas da autora na cidade do Rio de Janeiro. O texto, tratado como um exercício, traz uma escrita que transita a academia e a subjetividade de quem escreve, trazendo um recorte do DA que contempla o tempo, as imagens, os encontros inusitados com cenas cotidianas registradas em imagens que narram, junto ao texto, a experiência de afetar e ser afetado pela cidade.

Desta forma, o intuito dessa publicação é apresentar os esforços para se pensar e agir por meio de correspondências entre o design e a antropologia, 
e outros campos do saber aqui aludidos, como a moda, produzindo-se práticas inovadoras de ensino por projeto, desenho coletivo e cocriação. Boa leitura!

As editoras.

\section{Referências}

CLARKE, A. (Ed.). Design Anthropology. Object culture in the 21st Century. Wien: Springer-Verlag, 2011.

GATT, C.; INGOLD, T. "From description to correspondence: Anthropology in real time". In: W. Gunn, T. Otto and R. Smith (Eds.) Design Anthropology: Juxtaposing theory and practice. London, Delhi, New York, Sydney: Bloomsbury, 2013, p. 139-158.

GUNN, W.; OTTO, T.; SMITH, R. C. (Eds.). Design Anthropology: theory and practice. London and New York: Bloomsbury, 2013.

GUNN, W.; DONOVAN, J. (Eds.). Design and Anthropology. Surrey and Burlington: Ashgate, 2012.

HALSE, J.; BRANDT, E.; CLARK, B.; BINDER, T. (Eds.). Rehearsing the future. Copenhagen: The Danish Design School Press, 2010.

INGOLD, T. Anthropology and/as education. London: Routledge, 2018.

INGOLD, T. The life of lines. London: Routledge, 2015.

INGOLD, T. Making: anthropology, archeology, art and architecture. London: Routledge, 2013a.

INGOLD, T. Knowing From the Inside: Anthropology, Art, Architecture and Design. Research Project funded by the European Research Council and developed at Aberdeen University, Scotland, 2013b. https://www.abdn.ac.uk/research/kfi/

INGOLD, T. Being Alive. Essays on movement, knowledge and description. London: Routledge, 2011.

INGOLD, T. Lines: a brief history. London: Routledge, 2007.

INGOLD, T; LUCAS, R. “The 4 A's (Anthropology, Archaeology, Art and Architecture): Reflections on a Teach and Learning Experience”, 2007.

INGOLD, T.; HALLAM, E. Creativity and cultural improvisation. Oxford, New York: Berg, 2007.

INGOLD, T. The Perception of the Environment: essays on livelihood, dwelling and skill. London: Routledge, 2000.

LATOUR, B. "A Cautious Prometheus? A Few Steps Toward a Philosophy of Design (With Special Attention to Peter Sloterdijk)". Keynote lecture for the Networks of Design meeting of the Design History Society, Falmouth, Cornwall, 2008. 
MARCUS, G. E.; RABINOW, P. Designs for an anthropology of the contemporary. Durham and London: Duke University Press, 2008.

PAPANEK, Victor. Arquitectura e Design - Ecologia e Ética. Lisboa: Edições 70, 2002. 284p.

PAPANEK, Victor. Design for the Real World: Human Ecology and Social Change. New York, Pantheon Books, 1971. 60p.

SMITH, R.; VANGKILDE, K.; KJAERSGAARD, M.; HALSE, J.; BINDER, T. (Eds.) (Eds.). Design Anthropological Futures. London, New York: Bloomsbury, 2016.

WASSON, C. "Ethnography in the field of design". In: Human Organization, vol. 59, n. 4. Society for Applied Anthropology, 2000, p. 377- 388.

DOI: $10.12957 /$ arcosdesign.2018.47514

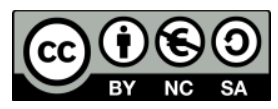

A Revista Arcos Design está licenciada sob uma licença Creative Commons Atribuição - Não Comercial - Compartilha Igual 3.0 Não Adaptada. 